\section{The Auroral Spectrum}

THE exact mode of excitation of the lines and bands in the auroral spectrum is not yet fully understood. Many attempts have been made to explain the spectrum, but none has been convincing ${ }^{1,2,8}$. The purpose of the present note is to offer a simple explanation of the auroral spectrum on the lines of the theory of emission of similar lines and bands from the luminescent layer of the night sky postulated by me some time ago ${ }^{4,5}$. Besides similarities, there are also differences between the two spectra; but these are to be traced mostly to the difference in the atmospheric heights at which the two phenomena originate: the height of the most frequent occurrence of aurora is
between 90 and $120 \mathrm{~km}$. (regions $D$ and $E$ of the ionosphere), and the between 90 and $120 \mathrm{~km}$. (regions $D$ and $E$ of the ionosphere), and the
height of the luminescent layer of the night sky is $200-400 \mathrm{~km}$. (region height of the luminescent layer of the night sky is $200-400 \mathrm{~km}$. (region
$F$ of the lonosphere). The collisional frequency of the atmospheric particles, which affects the emission of the spectra, is about two orders higher in the lower than in the higher region.

The primary cause of excitation of the auroral spectrum is bombardment by charged particles shot off from the sun. The spectrum consists of band systems of molecular nitrogen and certain lines of a tomic oxygen., These are, in order of prominence : flrst negative bands, $\mathrm{N}_{\mathbf{2}}+\left(A^{\prime} \rightarrow \mathcal{X}^{\prime}\right)$; auroral green line $\lambda 5577 \mathrm{O}\left({ }^{\prime} S \rightarrow{ }^{1} D\right)$; flrst positive bands, $\mathrm{N}_{2}(B \rightarrow A)$; red auroral triplet, $\lambda 6300 \mathrm{O}\left({ }^{(} D \rightarrow{ }^{8} P\right)$; second positive bands faintly, $\mathrm{N}_{2}(C \rightarrow B)$ and Vegard-Kaplan bands very faintly, $\mathrm{N}_{2}(A \rightarrow X)$. In regard to the atomic oxygen lines, it is to be is photo-dissociated by absorption of solar ultra-violet radiation (Runge-Schumann continuum $^{6}$ ). Above $115 \mathrm{~km}$. the gas is almost completely dissociated; the dissociation extends down to $100 \mathrm{~km}$. where it is estimated to be 7 per cent. Bombardment by charged particles shot off from the sun might also dissociate oxygen molecules and push the dissociation down to lower levels.

The bombardment, however, cannot be the immediate cause of excitation of all the spectra. As is well known, the red and green auroral lines are not ordinarily excited in a discharge tube or when oxygen gas is bombarded by canal rays, though other lines of $O$ are excited. The reason for the difference between upper atmospheric spectra and the discharge tube spectra is the part which the walls of the tube play in promoting quick disappearance of the products of ionization, for example, electrons, ions and metastable particles. Th electrons and ions recombine mostly on the surface (rather than in the volume), which acts as the third body absorbing the excess of energy and momentum. The metastable particles also, as they find their way to the walls, deliver up their energy by collision to the glass surface glass wall, the products of bombardment persist; and by reacting glass wall, the products of bombardment persist; and by reacting not ordinarily obtained in discharge tubes. (In connexion with the not ordinarily obtained in discharge tubes. (In connexion with the above, I have recently put forward the hypothesis that active nitrogen is only $\mathrm{N}_{2}+$ ions produced by the discharge ${ }^{7}$. They persist in the afterglow vessel due to special conditioning of the walls, which prevents the neutralization of the electrons and ions on the surface. 'The con-
ditioning is probably caused by adsorption of nitrogen, which has very little electron affinity.)

It is necessary, therefore, to distinguish between that part of the auroral spectrum which is directly excited as a result of bombardmen and that which is excited as a result of the interaction of the product of the bombardment (ions and electrons) among themselves and/or
with the neutral particles. To the flrst category belongs the flrst with the neutral particles. To the flrst category belongs the flrst
negative bands due to $\mathrm{N}_{2}+$ and to the second the rest of the spectrum First negative bands. The bombarding particles directly ionize and at the same time, excite the $\mathrm{N}_{2}+$ ions produced to the $A^{\prime}$ state from which the first negative bands are emitted. That the bombardmen of the upper atmosphere produces ionization is shown by ionospheri observations in high latitudes ${ }^{8}$. During auroral displays the ionization increases in intensity and extends down to the radio-wave absorbing region $(D)$, which causes obliteration of radio echoes. Assuming an isothermal atmosphere and that the light intensity is proportional to the ionization, Vegard has calculated the intensity distribution with height due to the bombarding action of fast charged particles entering the atmosphere ${ }^{\circ}$. The calculated distribution curve is very similar to the observed distribution curve for a large class of auroras. The first negative bands are also easily produced in the laboratory by the bombardment of nitrogen gas by cathode rays ${ }^{10}$

First positive bands and the oxygen lines. The mode of excitation of these is the same as that which I have proposed in the night sky. Briefly, it is as follows: the oxygen atoms, on account of their high electron affinity, attach to themselves electrons and form negative ions. These react with $\mathrm{N}_{2}+$ ions as follows: $\mathrm{N}_{2}++\mathrm{O}^{-} \rightarrow \mathrm{N}_{3}$ (excited) $+\mathrm{O}$ (excited). From the excited nitrogen molecules and oxygen atoms, the first positive bands and the green and red radiations are emitted respe

Second positive bands. The above reaction cannot account for these bands on account of inadequate energy. That their mode of excitation must be different is also evident from the fact that the nitrogen molecules after emission are left in the vibration levels $0-4$ of the $B$-state. The observed first positive bands, however, originate from levels 8-11 of the same state. The faint emission of the second positive bands may be explained as due to the recombination of $\mathrm{N}_{2}+$ ions and electrons by a radiative process :

$$
\mathrm{N}_{2}+e \rightarrow \mathrm{N}_{2}(B)+h v .
$$

The cross-section of $\mathrm{N}_{2}$ for this reaction is, however, small, being only $10^{-12} \mathrm{~cm} .^{2}$ as compared with $10^{-8} \mathrm{~cm} .^{2}$ for the other process.

The enhancement of the flrst positive bands in the rare case of the so-called red auroras of type $B$ (arcs and draperies, of which the lower fringe reaching abnormally low heights of $65-70 \mathrm{~km}$. is tinged red) falls in the first category. The bombarding particles are slowe down by deep penetration and excite the nitrogen molecules by direct impact.

Effects of higher collisional frequency on the auroral spectrum. Faintness of $\nabla-K$ bands ; greater strength of the green $O$-line than the tive strengthening of the red 0-radiations on passing up an aurora streamer.
These effects distinguish the auroral spectrum from the night sky spectrum. They are easily explained if the long lives of the metal stable states from which they originate and the decrease of collisionafrequency with height are taken into account. In regions of high collisional frequency, the molecules or atoms in metastable states have a greater chance of coming down to a lower state by collision with other atmospheric particles than by radiation.

Sunlit aurora. The remarkable enhancements of the negative bands of $\mathrm{N}_{2}+$ and of the red oxygen lines in sunlit aurora have been the subject of much comment, but no explanation has yet been given. The explanation is, however, simple if similar enhancements in the case of the night sky spectrum taken during twilight - when the rays of the rising or the setting sun touch the high atmosphere-are considered. (Compare, for example, the spectrum of the former taken by Störmer ${ }^{11}$ and that of the latter by EIvey ${ }^{12}$.) The enhancement of the negative bands is due to production of $\mathrm{N}_{2}+$ ions in the $A^{\prime}$-state by absorption of $\lambda<661^{13}$. The enhancement of the red lines (as compared with the green line which is unaffected) is due to two factors oxygen atom for $\lambda<6392$ for excitation to the $1 D$-state is much higher than that for $\lambda<2972$ for excitation to the ${ }^{1} S$-state ; and secondly, the density of solar radiation in the first spectral region is about a the density of solar radiation in the first spectral region is about a
hundred times greater than that in the second. (The case of enhancement in night sky spectra during twilight is fully discussed by Ghosh ${ }^{5}$.) The subject-matter of the communication is being studied in fuller detail by Mr. S. N. Ghosh, my research scholar, and will shortly be published in the Proceedings of the National Institute of Sciences of India.

Wireless Laboratory

S. K. Mitra

University College of Science,

92 Upper Circular Road,

Feb. 13 .

1 Vegard, L., Geofys. Pub., 10, No. 4 (1933).

2 Ta-You Wu, Proc. Ind. Acad. Sci. (Bangalore), 18, 40 (1943).

${ }^{3}$ Chapman, S., Phil. Mag., 23, 657 (1937).

Mitra, S. K., Science and Culture (Calcutta), 9, 46 (1943) ; Nature, $155,786(1945)$.

${ }^{5}$ Ghosh, S. N., Proc. Nat. Inst. Sci. (Calcutta), 9, 301 (1943)

Majumdar, R. C., Ind. J. Phys., 12, 75 (1938). Wulf, O. R., and paper).

" Mitra, S. K., Science and Culture (Calcutta), 9, 49 (1943) ; "Active Nitrogen-A New Theory" (Ind. Assoc. Cultiv. Sci., Calcutta, 1943).

s Appleton, E. V., Naismith, R., and Ingram, L. J., Phil. Trans. Roy. Soc., A, 236, 119 (1937)

- Vegard, L., Ann. Phys., 39, 111 (1912).

${ }^{10}$ Duncan, D. C., Astrophys. J., 62, 145 (1925).

11 Störmer, C., Nature, 142, 1034 (1938).

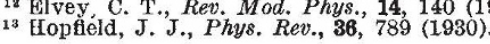

\section{Electronic Spectra of some Bent Triatomic Molecules}

THe purpose of this communication is to summarize the results obtained by us during recent years on the absorption electronic spectra of the $\mathrm{SO}_{2}, \mathrm{SeO}_{2}$ and $\mathrm{TeO}_{2}$ molecules. Whereas the size and the fundamental frequencies of $\mathrm{SO}_{2}$ are known accurately ${ }^{1}$, no corresponding mental frequencies of $\mathrm{SO}_{2}$ are known accurately, no corresponding data exist for $\mathrm{SeO}_{2}$ and $\mathrm{TeO}_{2}$. It has, however, been established that analogy, we may infer that this is also the case for tellurium dioxide. The electronic spectra of these three molecules were studied repeatedly ${ }^{3}$, but a detailed analysis was given only for the $b$ and 0

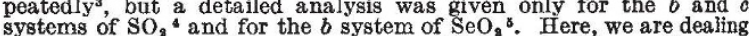
with the $a$ system of $\mathrm{SO}_{2}$, the $c$ system of $\mathrm{SeO}_{2}$ and with a preliminary investigation of the $\mathrm{TeO}_{2}$ bands.

$\mathrm{SO}_{2}$.- The $a$ system of $\mathrm{SO}_{2}$ was observed in absorption between 2000 and $2400 \mathrm{~A}$. under widely varied experimental conditions of temperature and pressure $\left(200^{\circ}-700^{\circ} \mathrm{K}\right.$.; $0.5 \mathrm{~mm} .-100 \mathrm{~mm}$. mercury pressure) and with an absorption path from $5 \mathrm{~cm}$. to $100 \mathrm{~cm}$. The dispersion used was either 2 A. min. or 5 A. min. at 2200 A. Contrary to the statements of Price and simpson, we have shown that the prominent bands constitute several different progressions. They can be ftted into two separate electronic systems designated

$\alpha_{1} \ldots v_{1}^{\prime}=962 \mathrm{~cm}^{-1}, v_{2}^{\prime}=379 \mathrm{~cm}^{-1}, x^{\prime} v_{2}^{\prime}=2 \mathrm{~cm}^{-1}, v_{0}=42,170 \mathrm{~cm}^{-1}$
$\alpha_{2} \ldots v_{1}^{\prime}=775 \mathrm{~cm} \cdot{ }^{-1}, v_{2}^{\prime}=375 \mathrm{~cm}^{-1}$,

The bands of the $\alpha_{1}$ system appear to be of the perpendicular type with $K$ structure well defined and degraded towards the red. The analysis gives $C^{\prime}=0.9 \mathrm{~cm} .^{-1}$ as compared with $C^{\prime \prime}=1.6 \mathrm{~cm}^{-1}$. The sponding sub-bands are slightly degraded towards the red and that the convergence is slow. If so, we have a case already discussed by Metropolis ${ }^{\circ}$ and related to $\triangle \alpha<0$ and $\Delta r \cong 0$, with $B^{\prime} \cong B^{\circ}$. From Fig. 2 of the paper referred to we can conclude

decreased by about $20^{\circ}$, while $r_{\theta}$ has increased a little. There are only two allowed transitions giving perpendicular to system ( $\lambda_{\max }=3700 \mathrm{~A}$.). Thus the $\alpha_{1}$ system must ccrrespond to $1 a_{2} \rightarrow 4 b_{2}$ transition. We must point out that this attribution does not agree quantitatively with Mulliken's scheme of $\mathrm{SO}_{\mathrm{s}}$ levels. than a qualitative meaning

We have not succeeded in analysing the fine structure of the $a_{2}$ system. Perhaps it could be correlated with $3 b_{2} \rightarrow 5 a_{1}$, as proposed even modify the order of the electronic terms in Mulliken's scheme 